\title{
EXTRATOS HEXÂNICOS DE FUMO, ALHO E PIMENTA-ROXA SOBRE FRANKLINIELLA SCHULTZEI (THYSANOPTERA: THRIPIDAE)
}

\author{
Vando Miossi Rondelli' \\ Vagner Tebaldi de Queiroz ${ }^{2}$ \\ Adilson Vidal Costa ${ }^{3}$ \\ Natália Assis Guedes ${ }^{4}$ \\ Priscila Stinguel ${ }^{5}$ \\ Marcel Oliveira Tiburcio ${ }^{6}$ \\ Kharen Priscila de Oliveira Silva Salomão ${ }^{7}$ \\ Marcos Américo da Silva ${ }^{8}$ \\ Dirceu Pratissoli ${ }^{9}$
}

Resumo: O tomateiro é uma das hortaliças mais importantes, no entanto o ataque de pragas, como o tripes, Frankliniella schultzei, pode causar prejuízos a essa cultura, principalmente por transmitir o complexo de vírus denominados de vira-cabeça do tomateiro. Contudo, os extratos de plantas podem ser uma alternativa no manejo de pragas. Assim, o objetivo foi avaliar o potencial inseticida de extratos hexânicos de fumo, alho e pimenta-roxa sobre F. schultzei. Esses extratos na concentração de $500 \mu \mathrm{g} \mathrm{mL}^{-1}$ foram testadas sobre ninfas de segundo ínstar de F. schultzei. O extrato hexânico de fumo foi o que causa maior mortalidade (28,1\%). Assim, nenhum dos extratos hexânicos avaliados neste trabalho causa mortalidade satisfatória sobre ninfas de F. schultzei, na concentração de $500 \mu \mathrm{g} \mathrm{mL}^{-1}$.

Palavras-chave: Nicotiana tabacum; Allium sativum; Capsicum chinense; Controle alternativo; Tomateiro.

\footnotetext{
${ }^{1}$ Agronomia/Universidade Federal de Rondônia, Brasil. E-mail: vando.rondelli@unir.br.

${ }^{2}$ Química Licenciatura/Universidade Federal do Espírito Santo, Brasil. E-mail: vagnertq@gmail.com.

${ }^{3}$ Química Licenciatura/Universidade Federal do Espírito Santo, Brasil. E-mail: avcosta@hotmail.com.

${ }^{4}$ Química Licenciatura/Universidade Federal do Espírito Santo, Brasil. E-mail: nataliaassisg@hotmail.com.

${ }^{5}$ Agronomia/Universidade Federal do Espírito Santo, Brasil. E-mail: pri_stinguel@hotmail.com.

${ }^{6}$ Agronomia/Universidade Federal do Espírito Santo, Brasil. E-mail: marcel.ccaufes@gmail.com.

${ }^{7}$ Agronomia/Universidade Federal do Espírito Santo, Brasil. E-mail: kharensalomao@yahoo.com.br.

${ }^{8}$ Agronomia/Universidade Federal do Espírito Santo, Brasil. E-mail: marcosameic@gmail.com.

${ }^{9}$ Agronomia/Universidade Federal do Espírito Santo, Brasil. E-mail: dirceu.pratissoli@gmail.com.
} 